\title{
P4HB: A novel diagnostic and prognostic biomarker for bladder carcinoma
}

\author{
YUCAI WU ${ }^{1-4^{*}}$, YIJI PENG ${ }^{1-4^{*}}$, BAO GUAN ${ }^{1-4}$, ANBANG HE ${ }^{1-4}$, KUNLIN YANG $^{1-4}$, \\ SHIMING HE ${ }^{1-4}$, YANQING GONG ${ }^{1-4}$, XUESONG LI ${ }^{1-4}$ and LIQUN ZHOU ${ }^{1-4}$ \\ ${ }^{1}$ Department of Urology, Peking University First Hospital; ${ }^{2}$ Institute of Urology, Peking University; \\ ${ }^{3}$ National Urological Cancer Center; ${ }^{4}$ Urogenital Diseases (Male) Molecular Diagnosis and Treatment Center, \\ Peking University, Xicheng, Beijing 100034, P.R. China
}

Received May 30, 2020; Accepted October 23, 2020

DOI: $10.3892 / 01.2020 .12356$

\begin{abstract}
Prolyl 4-hydroxylase, beta polypeptide (P4HB) protein is an endoplasmic reticulum (ER) molecular chaperone protein and has been reported to be overexpressed in multiple tumor types. However, the role of P4HB in bladder cancer (BLCA) has not yet been elucidated. The aim of the present study was to investigate the prognostic value of $\mathrm{P} 4 \mathrm{HB}$ and the association between clinicopathological characteristics and P4HB in BLCA. P4HB expression levels were assessed through The Cancer Genome Atlas (TCGA) and Gene Expression Omnibus (GEO) databases, and validated by reverse transcription-quantitative polymerase chain reaction (RT-qPCR) and western blot analysis in BLCA tissues and cells. A total of 69 pairs of tumor and normal samples were used to analyze the expression of $\mathrm{P} 4 \mathrm{HB}$ via immunohistochemical staining. A co-expression network and functional enrichment analyses were conducted to investigate the biological function of P4HB in BLCA. The protein-protein interaction (PPI) network was constructed by Search Tool for the Retrieval of Interacting Genes. The results showed that P4HB was highly
\end{abstract}

Correspondence to: Dr Yanqing Gong or Dr Xuesong Li, Department of Urology, Peking University First Hospital, 8 Xishiku Street, Xicheng, Beijing 100034, P.R. China

E-mail: yqgong@bjmu.edu.cn

E-mail: pineneedle@sina.com

${ }^{*}$ Contributed equally

Abbreviations: BLCA, bladder cancer; $\mathrm{P} 4 \mathrm{HB}$, prolyl 4-hydroxylase, beta polypeptide; TCGA, The Cancer Genome Atlas; GEO, Gene Expression Omnibus; RT-qPCR, reverse transcription-quantitative polymerase chain reaction; IHC, immunohistochemistry; OS, overall survival; RFS, recurrence-free survival; ER, endoplasmic reticulum; GEPIA, gene expression profiling interactive analysis; GO, gene ontology; KEGG, Kyoto Encyclopedia of Genes and Genomes; GSEA, gene set enrichment analysis; PPI, protein-protein interaction; NES, normalized enrichment score

Key words: prolyl 4-hydroxylase, beta polypeptide, bladder cancer, diagnostic, prognostic marker, ER stress expressed in BLCA cells and tissues. The area under the curve value for P4HB expression to discriminate between tumor and normal tissues was up to 0.888 (95\% CI: 0.801-0.975; $\mathrm{P}<0.001)$ and 0.881 (95\% CI: $0.825-0.937$; $\mathrm{P}<0.001)$ in TCGA database and our database, respectively. Furthermore, the expression level of $\mathrm{P} 4 \mathrm{HB}$ was an independent risk factor for overall survival (OS) and recurrence-free survival (RFS) by univariate and multivariate analyses. Kaplan-Meier survival analysis demonstrated that high P4HB expression was associated with low OS and RFS. Pathway enrichment analysis suggested that $\mathrm{P} 4 \mathrm{HB}$ was involved in protein processing in the endoplasmic reticulum (ER), including $\mathrm{N}$-glycan modification and protein metabolic processes responding to ER stress. PPI analysis revealed that the potential targets of P4HB were mainly involved in posttranslational protein modification and response to ER stress. In conclusion, the expression level of P4HB aid in identifying patients with early-stage BLCA and predicting the prognosis of BLCA. Therefore, P4HB may be a novel diagnostic and prognostic biomarker for BLCA.

\section{Introduction}

Bladder cancer (BLCA) is the fourth most common malignancy in males and it has high incidence and mortality rates despite improvements in its management over the past decade $(1,2)$. Non-muscle invasive bladder cancer is the most common type of BLCA because its low progression rates lead to longer patient survival times (3). However, 25-30\% of bladder tumors are found to be muscle invasive at the time of diagnosis and these patients often have a poor prognosis despite optimal treatment $(4,5)$. The prognosis of BLCA is determined by the initial tumor stage, but patients do not show specific symptoms in the early stages of BLCA. Therefore, identifying promising early molecular markers has an enormous application potential for monitoring the onset of malignant tumors and improving the clinical strategies for managing BLCA.

$\mathrm{P} 4 \mathrm{HB}$, also known as protein disulfide-isomerase; is a multifunctional protein that catalyzes the formation, breakage and rearrangement of disulfide bonds. It acts as a molecular chaperone that aids in ameliorating misfolded proteins in response to ER stress (6). In addition, upregulation of P4HB has been reported in certain cancer types and overexpression 
of $\mathrm{P} 4 \mathrm{HB}$ may promote the progression of malignant tumors, including gastric cancer, clear cell renal cell carcinoma and colon cancer (7-9). P4HB inhibition has been associated with chemosensitivity in glioblastoma multiforme via the endoplasmic reticulum stress response pathways $(10,11)$. In addition, P4HB-knockdown sensitized glioblastoma to radiotherapy by leading to ER stress and downregulating RAD51 gene expression (12). However, little is known regarding the association between $\mathrm{P} 4 \mathrm{HB}$ and BLCA. The present study therefore analyzed the prognostic value of $\mathrm{P} 4 \mathrm{HB}$ and the association between clinicopathological characteristics and $\mathrm{P} 4 \mathrm{HB}$ in BLCA.

\section{Materials and methods}

Tissue samples. A total of 69 BLCA tissues and adjacent tissues were obtained from patients (age range, 37-91 years; mean age, 64.1 years) with BLCA at the Department of Urology, Peking University First Hospital who underwent radical cystectomy between January 2007 and November 2012. Patients who were lost to follow-up and had missing data were excluded. The database included a population of 44 males and 25 females and the pathological diagnosis of these patients was transitional cell carcinoma. The histological characteristics of these samples were evaluated by hematoxylin-eosin staining and confirmed by experienced urological pathologists. Fresh samples were fixed with $4 \%$ paraformaldehyde for $12-24 \mathrm{~h}$ at room temperature and then paraffin-embedded for immunohistochemistry (IHC). The present study was approved by the Biomedical Research Ethics Committee of Peking University First Hospital and written informed consent was obtained from all patients.

Cell culture. The cell lines (SV-HUC-1, T24, SW780 and 5637) were obtained from the American Type Culture Collection and cultured according to the manufacturer's protocols. The normal human urinary tract epithelial SV-HUC-1 cell line was cultured in F-12K medium (Gibco; Thermo Fisher Scientific, Inc.), while the T24 and SW780 cell lines were cultured in Dulbecco's modified Eagle's medium (Gibco; Thermo Fisher Scientific, Inc.) and the 5637 cells were cultured in RPMI-1640 (Gibco; Thermo Fisher Scientific, Inc.). All media contained $10 \%$ fetal bovine serum (Gibco; Thermo Fisher Scientific, Inc.) and $1 \%$ penicillin G-streptomycin (Sigma-Aldrich; Merck KGaA). The cell cultures were maintained as a monolayer culture in a humidified atmosphere containing $5 \% \mathrm{CO}_{2}$ at $37^{\circ} \mathrm{C}$.

Reverse transcription-quantitative polymerase chain reaction $(R T-q P C R)$. Total RNA was extracted from tissue samples or cell lines using TRIzol reagent (Invitrogen; Thermo Fisher Scientific Inc.) and used according to the manufacturer's protocol. cDNA was generated using reverse transcription with the following conditions: $42^{\circ} \mathrm{C}$ for $15 \mathrm{~min}$ and $95^{\circ} \mathrm{C}$ for $3 \mathrm{~min}$ (TansGen).RT-qPCR was performed using the 7500Fluorescent Quantitative PCR System (Applied Biosystems; Thermo Fisher Scientific, Inc.), and GAPDH was used for normalization. The primer sequences used in the present study were as follows: P4HB forward, 5'-GGCTATCCCACC-ATCAAGTTC-3' and reverse, 5'-TCACGATGTCATCAGCCTCTC-3'; GAPDH forward, 5'-GGAGCGAGATCCCTCCAAAAT-3' and reverse, 5'-GGCTGTTGTC-ATACTTCTCATGG-3'. The PCR reaction was performed as follows: $94^{\circ} \mathrm{C}$ for $30 \mathrm{sec}$, then 40 cycles at $94^{\circ} \mathrm{C}$ for $5 \mathrm{sec}$ and at $60^{\circ} \mathrm{C}$ for $30 \mathrm{sec}$. All experiments were repeated at least three times. The relative expression level was calculated by using $2^{-\Delta \Delta \mathrm{Cq}}$ method (13).

$I H C$. The $5-\mu \mathrm{m}$ sections were cut from paraffin-embedded tissue samples, deparaffinized in xylene and rehydrated in a descending alcohol series. Next, the sections were heated $\left(120^{\circ} \mathrm{C}\right)$ for $20 \mathrm{~min}$ in citrate buffer $(10 \mathrm{mmol} / \mathrm{l}$; $\mathrm{pH} 6.0)$. Following treatment with $3 \%$ hydrogen peroxide to block the endogenous peroxidase activity, $10 \%$ normal goat serum was applied to reduce non-specific binding for $1 \mathrm{~h}$ at room temperature. Subsequently, the sections were incubated with primary rabbit anti-human $\mathrm{P} 4 \mathrm{HB}$ polyclonal antibody (1:10,000; cat. no. ab137110; Abcam) at $4^{\circ} \mathrm{C}$ overnight. The sections were then incubated with peroxidase-conjugated secondary antibodies (1:1,000; PV-9000; OriGene Technologies, Inc.) for $20 \mathrm{~min}$ at room temperature. All slides were washed three times and then the PowerVision ${ }^{\mathrm{TM}}$ two-step histostaining reagent and the 3,3-diaminobenzidine tetrahydrochloride substrate kit (Zhongshan Golden Bridge Biotechnology) were used to visualize the localization of the antigen according to the manufacturer's protocol. Two experienced independent pathologists examined all tumor slides by examining three random fields of view using a light microscope at x400 magnification (Olympus Corporation). The intensity of cellular staining was assigned a score of 0 (negative), 1 (weak), 2 (moderate) and 3 (strong). The proportion of stained tumor cells was scored as $0(0-5 \%)$, $1(6-25 \%), 2(26-50 \%), 3(51-75 \%)$ or $4(>75 \%)$. The multiplication of these two variables was calculated as the final score.

Western blotting. Total proteins were extracted from cell lines using NP-40 lysis buffer, quantified using BCA protein assay reagent (Pierce; Thermo Fisher Scientific, Inc.), and loaded onto $10 \%$ SDS gels $(20 \mu \mathrm{g} / \mathrm{lane})$. Proteins in the gel were transferred onto PVDF membranes (EMD Millipore) following electrophoresis. Following blocking at room temperature for $1 \mathrm{~h}$ with $5 \%$ skimmed milk, the membranes were incubated overnight at $4^{\circ} \mathrm{C}$ with an antibody against $\mathrm{P} 4 \mathrm{HB}$ (1:1,000; cat. no. ab137110; Abcam), followed by horseradish peroxidase-labeled anti-rabbit secondary antibody $(1: 5,000$; cat. no. sc-2004/sc-2005; Santa Cruz Biotechnology, Inc.). $\beta$-actin (1:1,000; cat. no. sc-8432; Santa Cruz Biotechnology, Inc.) and GAPDH (1:1,000; cat. no. sc-47724; Santa Cruz Biotechnology, Inc.) were used as the internal reference. Signals were detected by chemiluminescence using the ECL Western Blotting Detection Reagents (Beyotime Institute of Biotechnology).

In silico analysis of $\mathrm{P} 4 \mathrm{HB}$ using online datasets. Transcriptome and clinical data from the TCGA-BLCA datasets (including 410 patients with bladder carcinoma) were downloaded from UCSC XENA (https://xena.ucsc.edu/) (14). Affymetrix gene expression profiles were performed using Affymetrix Human Genome U133 Plus 2.0 (HG-U133 Plus_2.0) for two datasets (GSE42089 and GSE31189) and using Affymetrix Human Genome U133A Array (HG-U133A) for one dataset (GSE3167). The prognostic analyses were 
A

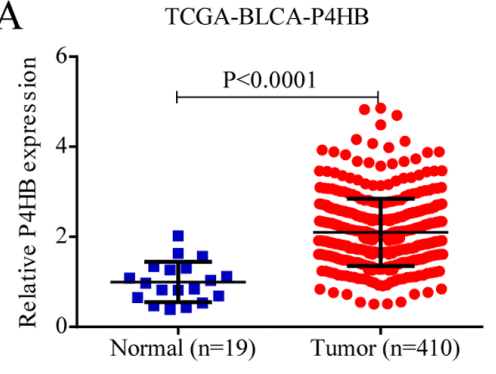

D

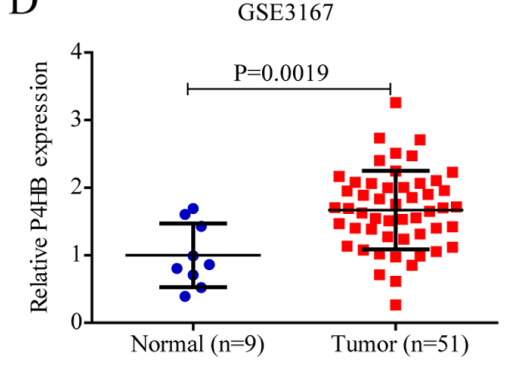

B

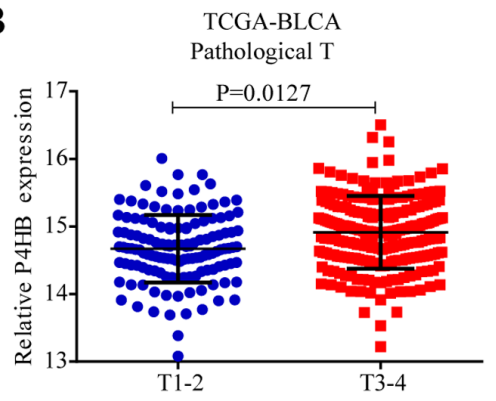

E

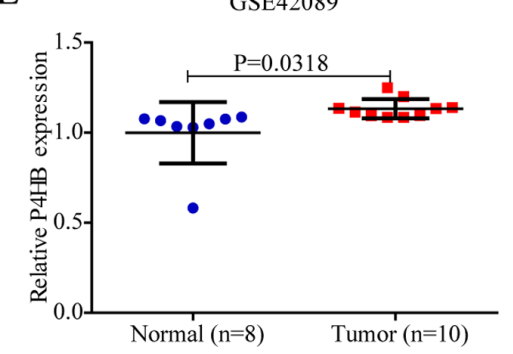

C

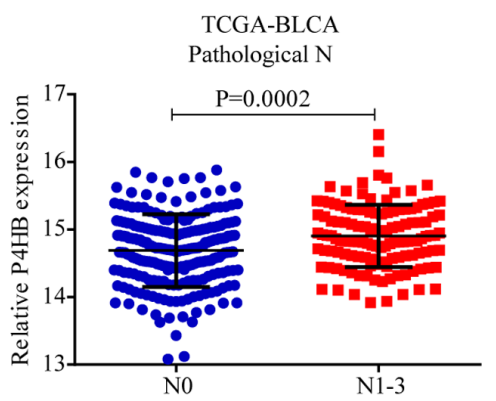

F

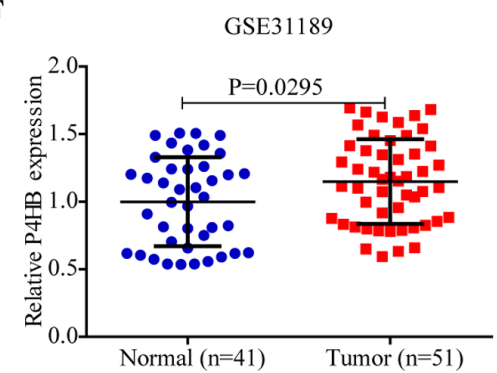

Figure 1. mRNA expression of P4HB in BLCA. (A) The mRNA expression of P4HB in TCGA clinical samples. P4HB expression among patients with BLCA with different pathological (B) T stages and (C) N stages. The mRNA expression of P4HB in the (D) GSE3167, (E) GSE42089 and (F) GSE31189datasets. P4HB, prolyl 4-hydroxylase, beta polypeptide; BLCA, bladder cancer; TCGA, The Cancer Genome Atlas.

performed by gene expression profiling interactive analysis (GEPIA; http://gepia.cancer-pku.cn/) (15). To investigate the mechanism of P4HB in BLCA, the co-expression genes of $\mathrm{P} 4 \mathrm{HB}$ were obtained using the limma R package (http://bioinf. wehi.edu.au/limma). The Pearson correlation coefficients between P4HB and protein-coding genes were calculated to determine the co-expression correlations (IPearson correlation coefficient $1>0.40$ and $\mathrm{P}<0.01)$. Gene Ontology (GO) analysis and Kyoto Encyclopedia of Genes and Genomes (KEGG) analysis were performed and visualized using the R package 'clusterProfiler' (16). The PPI network was constructed by Search Tool for the Retrieval of Interacting Genes (STRING; https://string-db.org/) (17) and modeled using Cytoscape software (Cytoscape_v3.6.1) (18). Gene set enrichment analysis (GSEA) was conducted to assess whether a predefined set of genes exhibited statistically significant, concordant differences according to P4HB expression by GSEA software (version 4.0.1; downloaded from http://software.broadinstitute. org/gsea/index.jsp) (19). The high expression group and the low expression group were defined according to the median mRNA level of P4HB. INormalized enrichment score (NES) $\mid>1$, false discovery rate (FDR) $<25 \%$ and nominal $\mathrm{P}<0.05$ were regarded as the cut-off criteria.

Statistical analysis. All experimental data were obtained from three independent experiments. SPSS version 24.0 (IBM Corp.) was used for statistical analyses. The difference between two sets of data was analyzed by Student's t-test or Mann-Whitney U test. One-way analysis of variance, followed by Dunnett's post hoc test, was applied to investigate the differences in P4HB expression between the normal cell line (SV-HUC-1) and the BLCA cell lines. Survival analysis was performed using the Kaplan-Meier estimator (log-rank test). Cox regression was conducted for univariate and multivariate analyses. The receiver operating characteristic curve (ROC) was plotted to evaluate the value of $\mathrm{P} 4 \mathrm{HB}$ expression as a predictor for early detection of BLCA. $\mathrm{P}<0.05$ was considered statistically significant.

\section{Results}

P4HB is upregulated in BLCA and associated with tumor stage. To investigate the expression of P4HB in BLCA, the mRNA expression of P4HB was analyzed in TCGA database. As shown in Fig. 1A, P4HB expression was higher in BLCA tissues than in normal tissues in TCGA $(\mathrm{P}<0.0001)$. Notably, the mRNA expression of P4HB was significantly higher at advanced pathological $\mathrm{T}$ stage $(\mathrm{P}<0.05)$ (Fig. 1B) and pathological $\mathrm{N}$ stage $(\mathrm{P}<0.001)$ (Fig. 1C). These data suggested that P4HB may be a promising biomarker for diagnosis and early detection in BLCA. The mRNA expression of P4HB was also verified in the GEO database, and analysis of the results of the GSE3167, GSE42089 and GSE31189 microarray datasets demonstrated that $\mathrm{P} 4 \mathrm{HB}$ was highly expressed in tumor tissues compared with normal tissues (Fig. 1D-F).

P4HB is highly expressed in bladder cancer cell lines and tissues. RT-qPCR was performed to investigate the mRNA expression of P4HB in BLCA cell lines and tissues. The results demonstrated that the mRNA expression level of P4HB was higher in tumor cell lines than in SV-HUC-1 cells (Fig. 2A). The mRNA expression of P4HB was increased in BLCA tissues, compared with paired normal mucosa. The average $\log 2$-fold-change of T/N was 1.67 (Fig. 2B). High mRNA expression of $\mathrm{P} 4 \mathrm{HB}$ was associated with a high pathological $\mathrm{T}$ stage $(\mathrm{P}<0.05$; Fig. $2 \mathrm{C})$ and $\mathrm{N}$ stage $(\mathrm{P}<0.05$; Fig. 2D). In addition, protein expression of $\mathrm{P} 4 \mathrm{HB}$ was upregulated in BLCA cell lines (Fig. 2E) and tissues (Fig. 2F). To better understand the expression of P4HB in BLCA tissues, an IHC assay was performed to investigate $\mathrm{P} 4 \mathrm{HB}$ protein expression levels in 
A
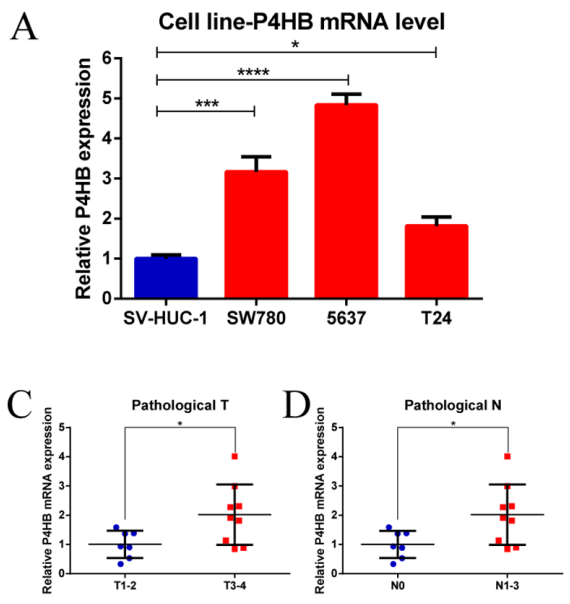

$\mathrm{E}$

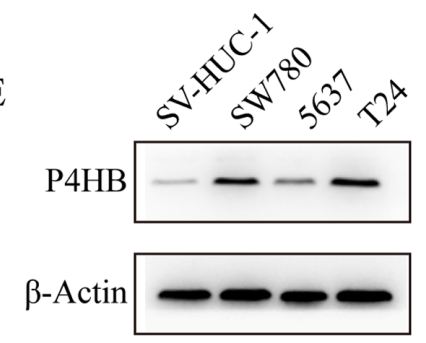

$\mathrm{G}$

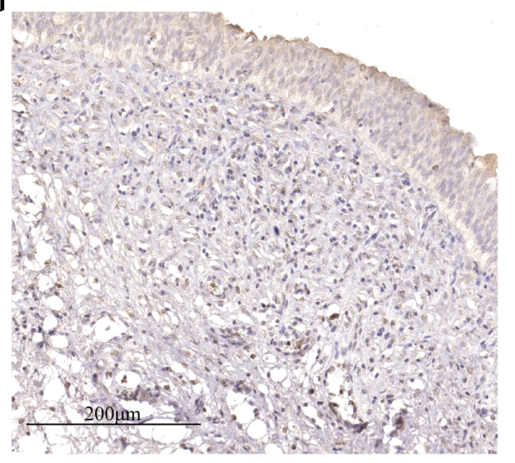

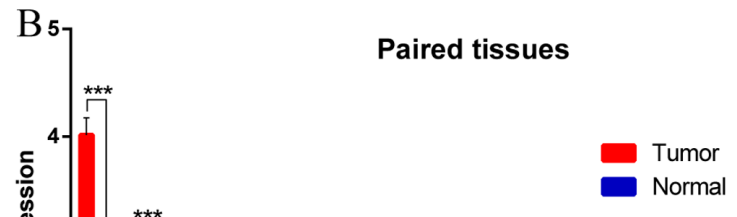

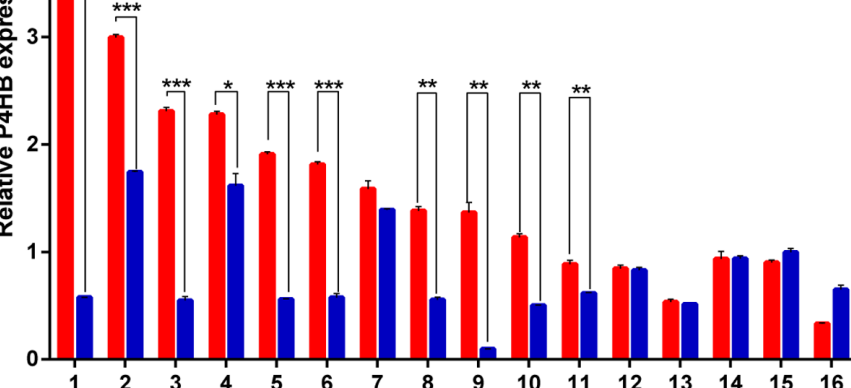

$\mathrm{F}$

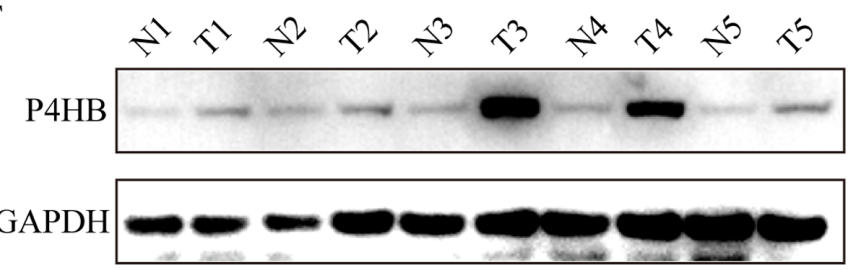

$\mathrm{H}$
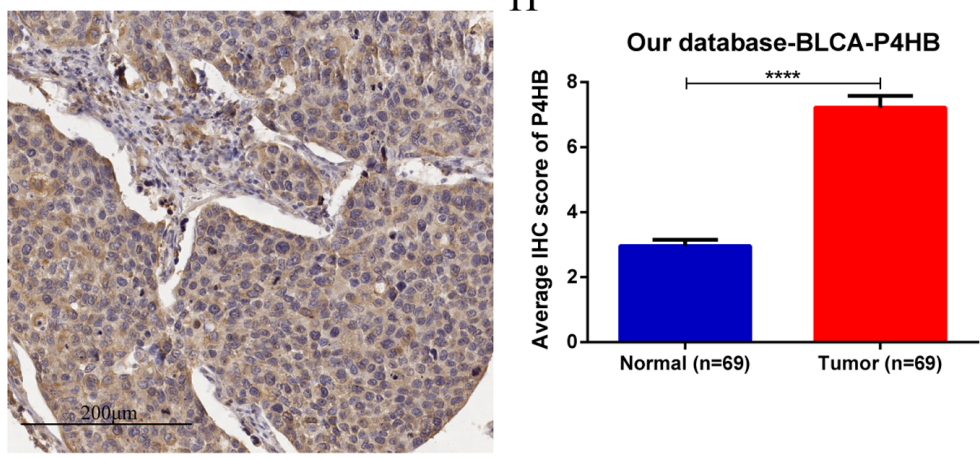

Figure 2. P4HB is highly expressed in bladder cancer cell lines and tissues. (A) P4HB mRNA expression in BLCA cell lines compared with normal uroepithelial SV-HUC-1 cells. (B) P4HB mRNA expression in 16 paired BLCA tissues and adjacent normal mucosa. P4HB expression among patients with BLCA at different pathological (C) T stages and (D) N stages. (E) P4HB protein expression in BLCA cell lines. (F) P4HB protein expression in 5 paired BLCA tissues. (G) IHC of BLCA tissues and adjacent normal tissues. The left image represents the normal mucosa and the right image represents the BLCA tissue. (H) The histogram represents the average IHC score of P4HB. ${ }^{*} \mathrm{P}<0.05 ;{ }^{* *} \mathrm{P}<0.01 ;{ }^{* * *} \mathrm{P}<0.001 ;{ }^{* * * * *} \mathrm{P}<0.0001$. P4HB, prolyl 4-hydroxylase, beta polypeptide; BLCA, bladder cancer; IHC, immunohistochemistry.

69 paired BLCA tissues and their adjacent normal tissues. The results demonstrated that $\mathrm{P} 4 \mathrm{HB}$ was highly expressed in BLCA tissues compared with paired normal mucosa (Fig. 2G). The average IHC score of T/N was 2.43 (Fig. 2H).

$P 4 H B$ is an early detection biomarker for BLCA. Considering the differential expression of P4HB between BLCA and normal mucosa tissues, ROC curves were generated to determine the diagnostic value of P4HB in TCGA database and our database. The ROC curve suggested that the AUC value for $\mathrm{P} 4 \mathrm{HB}$ expression to discriminate between tumor and normal was up to 0.888 (Fig. 3A; 95\% CI: 0.801-0.975; P<0.001) and 0.881 (Fig. 3B; 95\% CI: 0.825-0.937; $\mathrm{P}<0.001$ ) in TCGA and our database, respectively. In addition, the average IHC score of P4HB expression was significantly higher in advanced-stage and high-grade BLCA than in early-stage $(\mathrm{P}<0.01$; Fig. $3 \mathrm{C})$ and low-grade BLCA ( $\mathrm{P}<0.05$; Fig. 3D). Taken together, these results suggest that $\mathrm{P} 4 \mathrm{HB}$ may be an early detection biomarker for BLCA.

High P4HB expression predicts overall survival of patients with $B L C A$. A prospective study was performed to investigate the correlation between the OS time of BLCA and $\mathrm{P} 4 \mathrm{HB}$ expression. In addition to the tumor pathological N stage, P4HB expression was negatively correlated with OS in univariate analysis (95\% CI: 1.011-6.296; $\mathrm{P}=0.047$ ) in our database (Table I). Higher expression of P4HB exhibited a poorer OS based on Kaplan-Meier survival analysis $(\mathrm{P}<0.001$; Fig. 4A). In multivariate analysis, $\mathrm{N}$ stage $(95 \% \mathrm{CI}$ : 1.552-23.254; $\mathrm{P}=0.009)$ and $\mathrm{P} 4 \mathrm{HB}$ protein expression (95\% CI: 
Table I. Univariate analysis and multivariate analysis of overall survival of bladder cancer in our database.

\begin{tabular}{|c|c|c|c|c|}
\hline \multirow[b]{2}{*}{ Characteristic } & \multicolumn{2}{|c|}{ Univariate analysis } & \multicolumn{2}{|c|}{ Multivariate analysis } \\
\hline & $\mathrm{HR}(95 \% \mathrm{CI})$ & P-value & $\mathrm{HR}(95 \% \mathrm{CI})$ & P-value \\
\hline P4HB level, high vs. low & $2.523(1.011-6.296)$ & $0.047^{\mathrm{a}}$ & $3.303(1.240-8.798)$ & $0.017^{\mathrm{a}}$ \\
\hline Sex, male vs. female & $0.855(0.198-3.697)$ & 0.834 & & \\
\hline Age at initial diagnosis, $\geq 70$ vs. $<70$ years & $0.829(0.247-2.785)$ & 0.762 & & \\
\hline Pathological T, T3 + T4 vs. T1 + T2 & $2.438(0.991-5.999)$ & 0.052 & & \\
\hline Pathological N, N1-3 vs. N0 & $3.802(1.084-13.338)$ & $0.037^{\mathrm{a}}$ & $6.008(1.552-23.254)$ & $0.009^{\mathrm{a}}$ \\
\hline Histological grade, high vs. low & $1.958(0.884-4.333)$ & 0.098 & & \\
\hline
\end{tabular}

${ }^{\mathrm{a}} \mathrm{P}<0.05$. HR, hazard ratio; $\mathrm{CI}$, confidence interval.

A
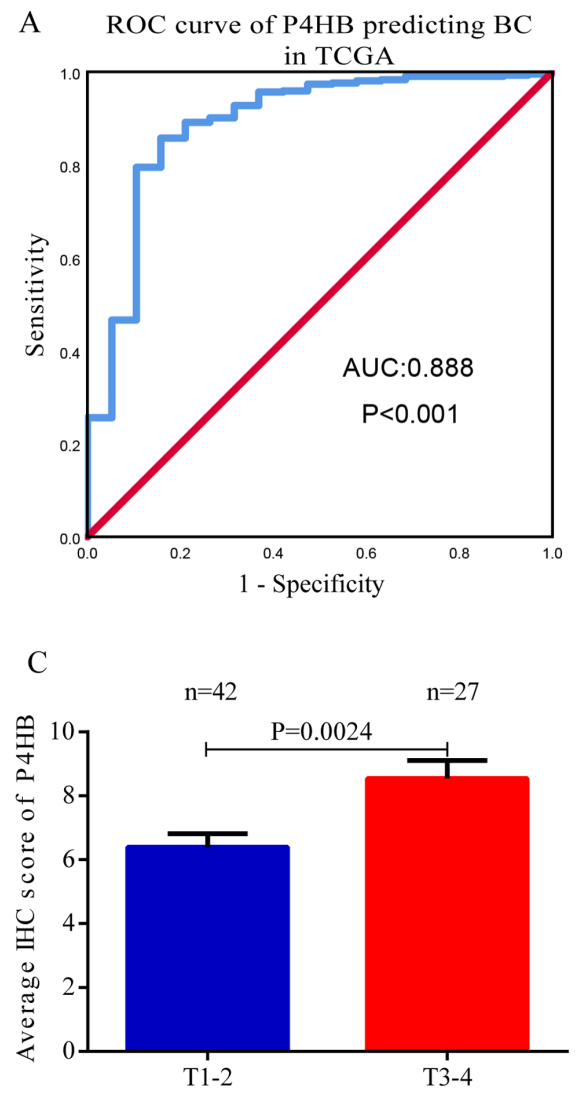

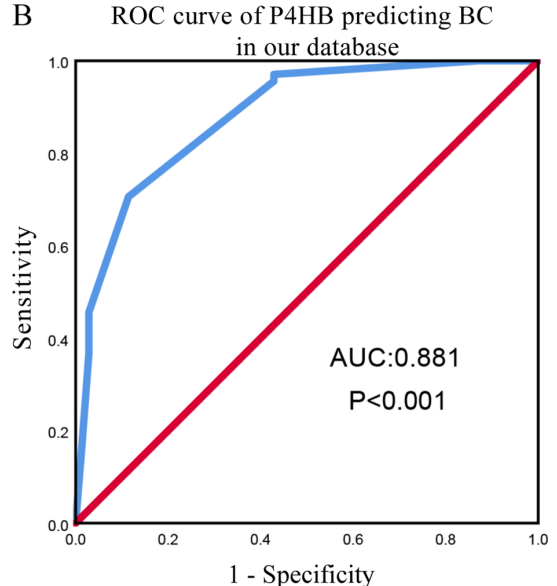

D

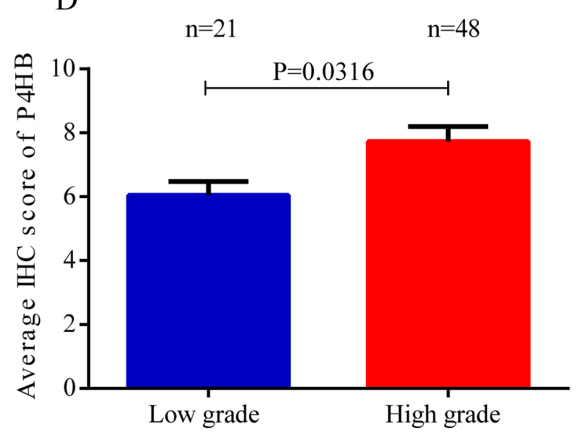

Figure 3. P4HB is an early detection biomarker for BLCA. ROC curve of P4HB expression to predict patients with BLCA in (A) TCGA database and (B) our database. Average immunohistochemistry scores of $\mathrm{P} 4 \mathrm{HB}$ at different (C) pathological stages and (D) histological grades. P4HB, prolyl 4-hydroxylase, beta polypeptide; BLCA, bladder cancer; ROC, receiver operating characteristic; TCGA, The Cancer Genome Atlas database.

1.240-8.798; $\mathrm{P}=0.017)$ were still correlated with a poorer OS, indicating that $\mathrm{P} 4 \mathrm{HB}$ may be an independent prognostic factor for patients with BLCA. Furthermore, the impact of clinicopathological parameters on patient survival in TCGA database was investigated. The mRNA expression of $\mathrm{P} 4 \mathrm{HB}$ remained an independent prognostic factor (Table SI), and high mRNA expression of P4HB was associated with a short OS time $(\mathrm{P}<0.01$; Fig. 4B). Considering that the pathological stage has a significant impact on patient survival, a nomogram containing IHC score, pathological $\mathrm{T}$ stage and $\mathrm{N}$ stage was constructed to predict the 5-year survival rate of patients with BLCA (Fig. 4C). The calibration curves for 5-year survival demonstrated that the predicted OS was close to the actual OS (Fig. 4D).

High $P 4 H B$ expression predicted recurrence-free survival in patients with BLCA. The correlation between the RFS of BLCA and $\mathrm{P} 4 \mathrm{HB}$ expression was further investigated. The results of IHC demonstrated that high pathological $\mathrm{N}$ stage and $\mathrm{P} 4 \mathrm{HB}$ protein levels were significantly associated with a poorer RFS by univariate and multivariate analyses (Table II). A similar result was obtained in TCGA database (Table SII). To determine whether $\mathrm{P} 4 \mathrm{HB}$ was a promising target for predicting RFS, Kaplan-Meier survival analysis was 
Table II. Univariate analysis and multivariate analysis of recurrence-free survival of bladder cancer in our database.

\begin{tabular}{|c|c|c|c|c|}
\hline \multirow[b]{2}{*}{ Characteristic } & \multicolumn{2}{|c|}{ Univariate analysis } & \multicolumn{2}{|c|}{ Multivariate analysis } \\
\hline & $\mathrm{HR}(95 \% \mathrm{CI})$ & P-value & $\mathrm{HR}(95 \% \mathrm{CI})$ & P-value \\
\hline P4HB level, high vs. low & $2.265(1.001-5.696)$ & $0.042^{\mathrm{a}}$ & $2.936(1.104-7.805)$ & $0.031^{\mathrm{a}}$ \\
\hline Sex, male vs. female & $0.932(0.216-4.031)$ & 0.925 & & \\
\hline Age at initial diagnosis, $\geq 70$ vs. $<70$ years & $0.735(0.218-2.479)$ & 0.620 & & \\
\hline Pathological T, T3 + T4 vs. T1 + T2 & $2.187(0.883-5.417)$ & 0.091 & & \\
\hline Pathological N, N1-3 vs. N0 & $4.199(1.181-14.922)$ & $0.027^{\mathrm{a}}$ & $6.318(1.629-24.504)$ & $0.008^{\mathrm{a}}$ \\
\hline Histological grade, high vs. low & $2.007(0.907-4.438)$ & 0.085 & & \\
\hline
\end{tabular}

${ }^{\mathrm{a}} \mathrm{P}<0.05$. HR, hazard ratio; $\mathrm{CI}$ : confidence interval.
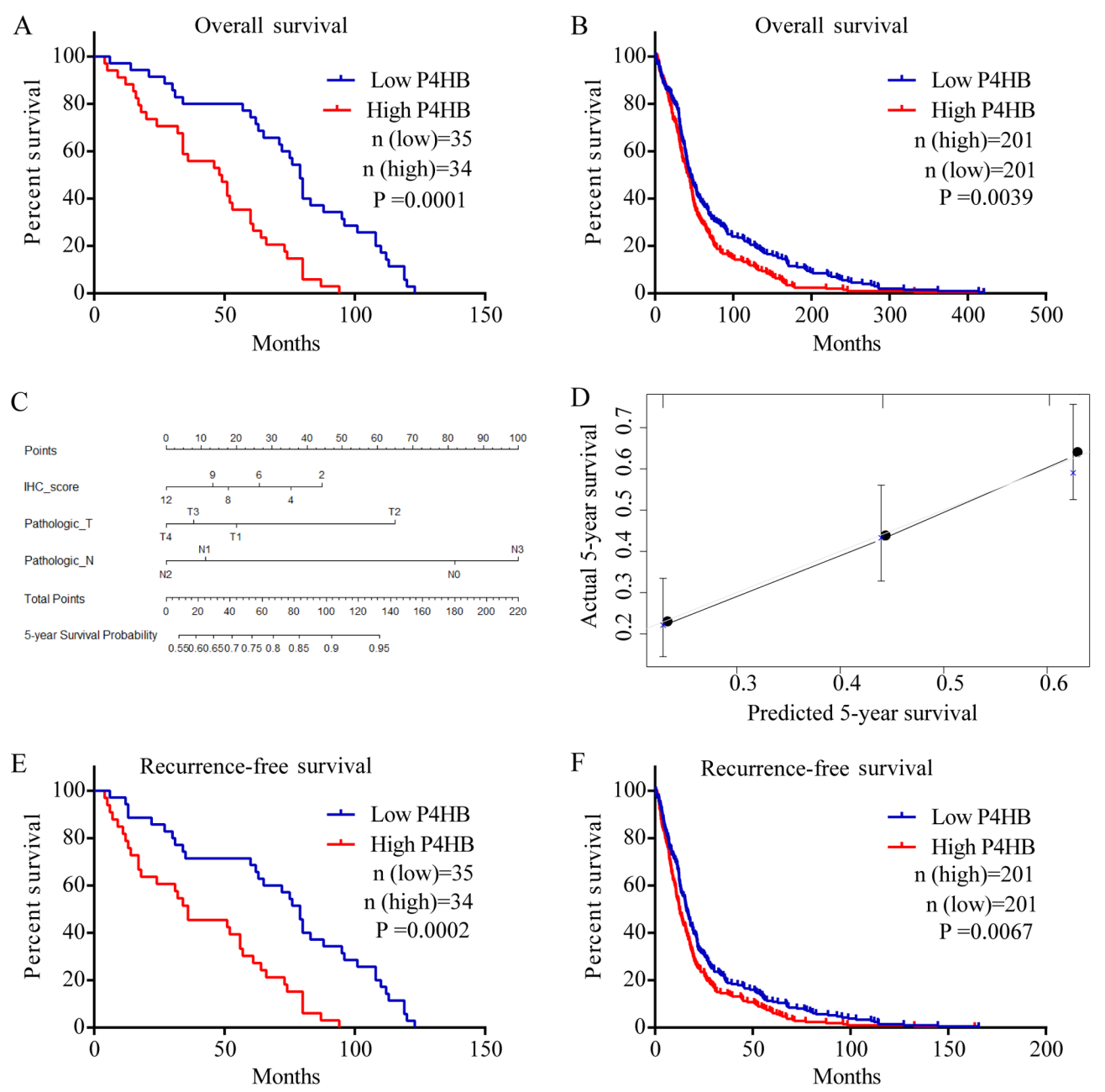

Figure 4. High P4HB expression was associated with poor survival in patients with BLCA. Kaplan-Meier survival curves for the OS of patients with BLCA based on P4HB expression in (A) our database and (B) TCGA database. (C) Nomogram to predict the 5-year OS of patients with BLCA. (D) Calibration plot evaluating the predictive accuracy of the nomogram for 5-year survival rate. Kaplan-Meier survival curves for the RFS of patients with BLCA based on P4HB expression in (E) our database and (F) TCGA database. P4HB, prolyl 4-hydroxylase, beta polypeptide; BLCA, bladder cancer; OS, overall survival; TCGA, The Cancer Genome Atlas database; RFS, recurrence-free survival.

conducted in our database and TCGA database. The results demonstrated that high expression of P4HB was associated with low RFS (Fig. 4E and F).

Functional prediction of $P 4 H B$ in BLCA by enrichment analysis. To investigate the biological function of P4HB in BLCA,
GO term enrichment analysis and KEGG pathway analysis were performed using the co-expression genes of P4HB. A total of 233 genes co-expressed with P4HB were selected from TCGA-BLCA database (Table SIII). Terms related to transferase activity, ribonucleoprotein complex binding, signal sequence binding and relevant member terms in ER were 
Table III. Functional enrichments in the protein-protein interactions network of prolyl 4-hydroxylase, beta polypeptide.

\begin{tabular}{lcl}
\hline Term description & FDR & \multicolumn{1}{c}{ Matching proteins in the network (labels) } \\
\hline Post-translational protein modification & $1.52 \mathrm{E}-09$ & $\begin{array}{l}\text { ALB, APOB, CALU, DNAJC3, HSP90B1, NUCB1, P4HB, } \\
\text { PDIA6, PRKCSH, RCN1 }\end{array}$ \\
& & $\begin{array}{l}\text { CALR, DNAJC3, ERO1L, HSP90B1, HSPA5, } \\
\text { OS9, P4HB, PDIA6 }\end{array}$
\end{tabular}

FDR, false discovery rate.
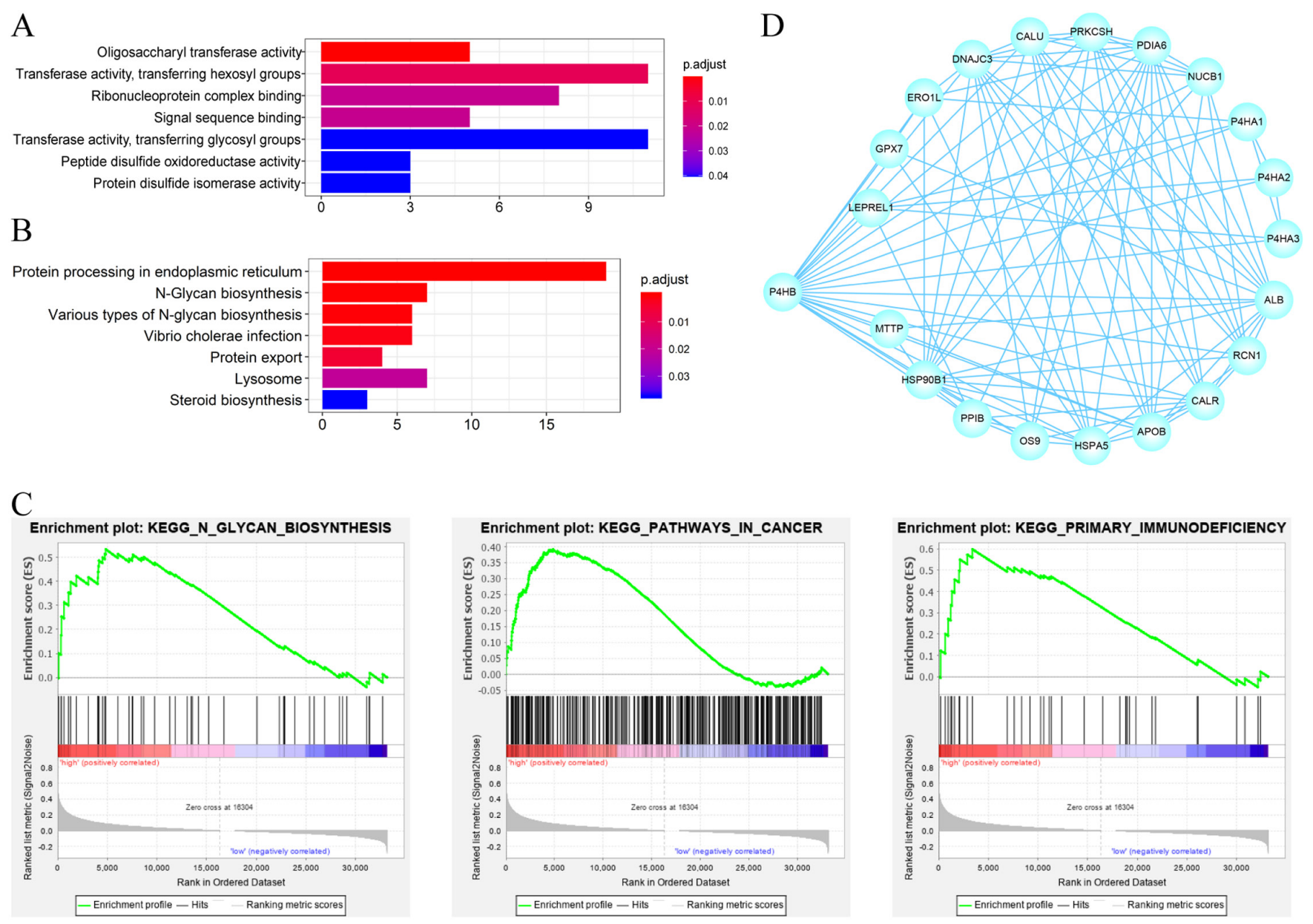

Figure 5. Functional prediction of P4HB in BLCA by enrichment analysis. (A) GO term and (B) KEGG pathway enrichment analysis results for genes co-expressed with P4HB in BLCA. (C) GSEA was performed in TCGA database according to the expression level of P4HB. The median expression of P4HB was selected as the cut-off value. (D) The protein-protein interaction network of P4HB and its potential targets. P4HB, prolyl 4-hydroxylase, beta polypeptide; BLCA, bladder cancer; GO, gene ontology; KEGG, Kyoto Encyclopedia of Genes and Genomes; GSEA, Gene set enrichment analysis; TCGA, The Cancer Genome Atlas database.

enriched (Fig. 5A and Table SIV). These terms were associated with carbohydrate metabolism and protein metabolism. Genes involved in protein processing in the ER, $\mathrm{N}$-glycan biosynthesis, various types of $\mathrm{N}$-glycan biosynthesis and other relevant pathways were also enriched (Fig. 5B and Table SV). In addition, GSEA in BLCA was performed by ranking the genes based on the expression of $\mathrm{P} 4 \mathrm{HB}$. High $\mathrm{P} 4 \mathrm{HB}$ expression was associated with several significant processes that promote tumorigenesis, including $\mathrm{N}$-glycan biosynthesis, pathways in cancer and primary immunodeficiency (Fig. 5C). These results indicated that P4HB may promote bladder tumorigenesis. PPI analysis revealed that the potential targets of $\mathrm{P} 4 \mathrm{HB}$ were mainly genes associated with posttranslational protein modification and response to ER stress (Fig. 5D and Table III). Taken together, these results implied that $\mathrm{P} 4 \mathrm{HB}$ may promote tumorigenesis by interfering with protein processing in the ER, including $\mathrm{N}$-glycan modification and protein metabolic processes responding to ER stress.

\section{Discussion}

P4HB, encoded by the P4HB gene on human chromosome $17 q 25$, is the $\beta$-subunit of prolyl-4-hydroxylase involved in 
antioxidant and detoxification reactions (20). P4HB may catalyze the oxidation, reduction and isomerization of disulfide bonds in the ER, which is essential to maintain homeostasis of the ER (20,21). Furthermore, P4HB acts as a chaperone and binds to misfolded proteins to prevent their excessive aggregation (22). The ER is an important organelle in eukaryotic cells that participates in a variety of intracellular metabolic and signal transduction pathways. Once ER homeostasis is disturbed, misfolded proteins accumulate, eventually leading to ER stress. The ER has formed self-protecting signal transduction pathways during evolution, including the unfolded protein response (UPR) of the ER, ER-associated degradation and autophagy to restore proteostasis. Furthermore, the ability to tolerate persistent ER stress enhances cancer cell survival, angiogenesis, aggressiveness, drug resistance and immunosuppression $(23,24)$.

As a potential therapeutic target, $\mathrm{P} 4 \mathrm{HB}$ has been found to be a novel diagnostic and prognostic marker in various cancer types, including colon, gastric and clear cell renal cell carcinoma $(7,8,25)$. Lovat et al $(26)$ demonstrated that overexpression of wild-type $\mathrm{P} 4 \mathrm{HB}$ in melanoma cells decreased the level of apoptosis in response to ER stress, which was beneficial to the survival of tumor cells. Furthermore, recent studies have demonstrated that $\mathrm{P} 4 \mathrm{HB}$ is overexpressed in gliomas and that $\mathrm{P} 4 \mathrm{HB}$ suppression sensitizes glioblastoma to chemotherapy and radiotherapy by abrogating ER stress-induced UPR signaling $(10,12,27)$. These results suggested the clinical importance of $\mathrm{P} 4 \mathrm{HB}$ in tumorigenesis and progression.

The present study investigated and confirmed the expression and clinical significance of P4HB in BLCA by analyses in TCGA, GEO and our clinical database. The results demonstrated that $\mathrm{P} 4 \mathrm{HB}$ expression was increased in BLCA tissues and cells. The expression of $\mathrm{P} 4 \mathrm{HB}$ was significantly correlated with tumor stage. Furthermore, based on immunohistochemical staining analysis, it was revealed that the expression of P4HB was an independent risk factor for OS and RFS. Furthermore, high expression of $\mathrm{P} 4 \mathrm{HB}$ was associated with low OS and RFS rates by Kaplan-Meier survival analysis in TCGA database and our database. These data indicated that P4HB may be a promising biomarker for predicting survival and tumor progression in patients with BLCA.

To better understand the oncogenic role of $\mathrm{P} 4 \mathrm{HB}$ in BLCA, functional prediction was performed using bioinformatics analysis. As indicated by the enrichment analyses, $\mathrm{P} 4 \mathrm{HB}$ may be involved in protein processes responding to ER stress and $\mathrm{N}$-glycan biosynthesis. It was reported that $\mathrm{N}$-glycans of certain proteins have a vital role in metastatic process, including epithelial-mesenchymal transition (EMT), migration, invasion/intravasation and extravasation of tumor cells (28). Inhibiting N-glycans markedly decreased receptor tyrosine kinase signaling in cancer cells and blocking N-glycan precursor biosynthesis may be a novel target for cancer therapy $(29,30)$. The target genes predicted by PPI analysis were mainly involved in posttranslational protein modification and protein processing in response to ER stress. The results of GSEA suggested that P4HB may enhance the development of BLCA by multiple significant processes, including N-glycan biosynthesis, pathways in cancer and primary immunodeficiency.
Although the expression profile of P4HB in BLCA has been identified in the present study, the oncogenic functions of $\mathrm{P} 4 \mathrm{HB}$ in BLCA need to be clarified in vitro and in vivo. Furthermore, the molecular mechanisms by which $\mathrm{P} 4 \mathrm{HB}$ functions need to be demonstrated in subsequent studies.

In summary, the results of the present study demonstrated that $\mathrm{P} 4 \mathrm{HB}$ may be a promising biomarker as it was overexpressed in BLCA and high expression of $\mathrm{P} 4 \mathrm{HB}$ was associated with a poor prognosis. Bioinformatics analysis revealed that $\mathrm{P} 4 \mathrm{HB}$ may be involved in $\mathrm{N}$-glycan biosynthesis and ER stress responses to promote BLCA tumorigenesis and progression. The detailed mechanisms of $\mathrm{P} 4 \mathrm{HB}$ in regulating BLCA and the potential association between $\mathrm{P} 4 \mathrm{HB}, \mathrm{N}$-glycans and ER stress require further investigation.

\section{Acknowledgements}

Not applicable.

\section{Funding}

The present study was funded by The National Key R\&D Program of China (grant no. 2019YFA09006001) and The National Natural Science Foundation of China (grant nos. 81772703 and 81972380).

\section{Availability of data and material}

The datasets used and/or analyzed during the current study are available from the corresponding author upon reasonable request. Publicly available datasets can be found in TCGA (https://portal.gdc.cancer.gov/) and GEO (https://www.ncbi. nlm.nih.gov/geo/) databases.

\section{Author contributions}

YW and YP designed the study, analyzed and interpreted the data, and drafted and critically revised the manuscript; $\mathrm{AH}$, KY and BG acquired the data and performed the statistical analysis; $\mathrm{SH}$ directed the research and performed the statistcal analysis; YG, XL and LZ interpreted the data and critically revised the manuscript for important intellectual content, provided administrative support, obtained funding and supervised the study. All authors read and approved the final manuscript.

\section{Ethics approval and consent to participate}

The present study was approved by the Biomedical Research Ethics Committee of Peking University First Hospital (Beijing, China), and written informed consent was obtained from all patients.

\section{Patient consent for publication}

Written consent for publication was obtained from all participants.

\section{Competing interests}

The authors declare that they have no competing interests. 


\section{References}

1. Siegel RL, Miller KD and Jemal A: Cancer statistics, 2019. CA Cancer J Clin 69: 7-34, 2019.

2. Antoni S, Ferlay J, Soerjomataram I, Znaor A, Jemal A and Bray F: Bladder cancer incidence and mortality: A global overview and recent trends. Eur Urol 71: 96-108, 2017.

3. Babjuk M, Burger M, Compérat EM, Gontero P, Mostafid AH, Palou J, van Rhijn BW, Rouprêt M, Shariat SF, Sylvester R, et al: European association of urology guidelines on non-muscle-invasive bladder cancer (TaT1 and carcinoma in situ) - 2019 Update. Eur Urol 76: 639-657, 2019.

4. Burger M, Catto JW, Dalbagni G, Grossman HB, Herr H, Karakiewicz P, Kassouf W, Kiemeney LA, La Vecchia C, Shariat S, et al: Epidemiology and risk factors of urothelial bladder cancer. Eur Urol 63: 234-241, 2013.

5. Alfred Witjes J,Lebret T, Compérat EM, Cowan NC, De Santis M, Bruins HM, Hernández V, Espinós EL, Dunn J, Rouanne M, et al: Updated 2016 EAU guidelines on muscle-invasive and metastatic bladder cancer. Eur Urol 71: 462-475, 2017.

6. Parakh S and Atkin JD: Novel roles for protein disulphide isomerase in disease states: A double edged sword? Front Cell Dev Biol 3: 30, 2015.

7. Zhang J, Guo S, Wu Y, Zheng ZC, Wang Y and Zhao Y: P4HB, a novel hypoxia target gene related to gastric cancer invasion and metastasis. BioMed Res Int 2019: 9749751, 2019.

8. Zhou Y, Yang J, Zhang Q, Xu Q, Lu L, Wang J and Xia W: P4HB knockdown induces human HT29 colon cancer cell apoptosis through the generation of reactive oxygen species and inactivation of STAT3 signaling. Mol Med Rep 19: 231-237, 2019.

9. Zhu Z, He A, Lv T, Xu C, Lin L and Lin J: Overexpression of $\mathrm{P} 4 \mathrm{HB}$ is correlated with poor prognosis in human clear cell renal cell carcinoma. Cancer Biomark 26: 431-439, 2019.

10. Sun S, Lee D, Ho AS, Pu JK, Zhang XQ, Lee NP, Day PJ, Lui WM, Fung CF and Leung GK: Inhibition of prolyl 4-hydroxylase, beta polypeptide (P4HB) attenuates temozolomide resistance in malignant glioma via the endoplasmic reticulum stress response (ERSR) pathways. Neuro Oncol 15: 562-577, 2013.

11. Lee D, Sun S, Zhang XQ, Zhang PD, Ho AS, Kiang KM, Fung CF, Lui WM and Leung GK: MicroRNA-210 and endoplasmic reticulum chaperones in the regulation of chemoresistance in glioblastoma. J Cancer 6: 227-232, 2015.

12. Liu Y, Ji W, Shergalis A, Xu J, Delaney AM, Calcaterra A, Pal A, Ljungman M, Neamati $\mathrm{N}$ and Rehemtulla A: Activation of the unfolded protein response via inhibition of protein disulfide isomerase decreases the capacity for DNA repair to sensitize glioblastoma to radiotherapy. Cancer Res 79: 2923-2932, 2019.

13. Livak KJ and Schmittgen TD: Analysis of relative gene expression data using real-time quantitative PCR and the 2(-Delta Delta C(T)) method. Methods 25: 402-408, 2001.

14. Goldman MJ, Craft B, Hastie M, Repečka K, McDade F, Kamath A, Banerjee A, Luo Y, Rogers D, Brooks AN, et al: Visualizing and interpreting cancer genomics data via the Xena platform. Nat Biotechnol 38: 675-678, 2020.

15. Tang Z, Li C, Kang B, Gao G, Li C and Zhang Z: GEPIA: A web server for cancer and normal gene expression profiling and interactive analyses. Nucleic Acids Res 45: W98-W102, 2017.
16. Yu G, Wang LG, Han Y and He QY: clusterProfiler: An R package for comparing biological themes among gene clusters. OMICS 16: 284-287, 2012.

17. Szklarczyk D, Franceschini A, Wyder S, Forslund K, Heller D, Huerta-Cepas J, Simonovic M, Roth A, Santos A, Tsafou KP, et al: STRING v10: Protein-protein interaction networks, integrated over the tree of life. Nucleic Acids Res 43: D447-D452, 2015.

18. Shannon P, Markiel A, Ozier O, Baliga NS, Wang JT, Ramage D, Amin N, Schwikowski B and Ideker T: Cytoscape: A software environment for integrated models of biomolecular interaction networks. Genome Res 13: 2498-2504, 2003.

19. Subramanian A, Kuehn H, Gould J, Tamayo P and Mesirov JP: GSEA-P: A desktop application for Gene Set Enrichment Analysis. Bioinformatics 23: 3251-3253, 2007.

20. Hatahet F and Ruddock LW: Substrate recognition by the protein disulfide isomerases. FEBS J 274: 5223-5234, 2007.

21. Freedman RB, Hirst TR and Tuite MF: Protein disulphide isomerase: Building bridges in protein folding. Trends Biochem Sci 19: 331-336, 1994

22. Wilson R, Lees JF and Bulleid NJ: Protein disulfide isomerase acts as a molecular chaperone during the assembly of procollagen. J Biol Chem 273: 9637-9643, 1998.

23. Moon HW, Han HG and Jeon YJ: Protein quality control in the endoplasmic reticulum and cancer. Int J Mol Sci 19: 3020, 2018.

24. Cubillos-Ruiz JR, Bettigole SE and Glimcher LH: Tumorigenic and immunosuppressive effects of endoplasmic reticulum stress in cancer. Cell 168: 692-706, 2017.

25. Xie L, Li H, Zhang L, Ma X, Dang Y, Guo J, Liu J, Ge L, Nan F, Dong H, et al: Autophagy-related gene P4HB: A novel diagnosis and prognosis marker for kidney renal clear cell carcinoma. Aging (Albany NY) 12: 1828-1842, 2020.

26. Lovat PE, Corazzari M, Armstrong JL, Martin S, Pagliarini V, Hill D, Brown AM, Piacentini M, Birch-Machin MA and Redfern CP: Increasing melanoma cell death using inhibitors of protein disulfide isomerases to abrogate survival responses to endoplasmic reticulum stress. Cancer Res 68: 5363-5369, 2008.

27. Peng Z, Chen Y, Cao H, Zou H, Wan X, Zeng W, Liu Y, Hu J, Zhang N, Xia Z, et al: Protein disulfide isomerases are promising targets for predicting the survival and tumor progression in glioma patients. Aging (Albany NY) 12: 2347-2372, 2020.

28. Oliveira-Ferrer L, Legler K and Milde-Langosch K: Role of protein glycosylation in cancer metastasis. Semin Cancer Biol 44: 141-152, 2017.

29. Contessa JN, Bhojani MS, Freeze HH, Ross BD, Rehemtulla A and Lawrence TS: Molecular imaging of N-linked glycosylation suggests glycan biosynthesis is a novel target for cancer therapy. Clin Cancer Res 16: 3205-3214, 2010.

30. Contessa JN, Bhojani MS, Freeze HH, Rehemtulla A and Lawrence TS: Inhibition of N-linked glycosylation disrupts receptor tyrosine kinase signaling in tumor cells. Cancer Res 68: 3803-3809, 2008.

This work is licensed under a Creative Commons Attribution-NonCommercial-NoDerivatives 4.0 International (CC BY-NC-ND 4.0) License. 